\title{
Genetic influence on tonic immobility in chickens*
}

\author{
GORDON G. GALLUP, JR. \\ Tulane University, New Orleans, Louisiana 70118
}

\begin{abstract}
Production Red chickens were selectively bred on the basis of having shown prolonged or brief immobility reactions at 21 days of age. After only one generation, there was sufficient separation between offspring to conclude that tonic immobility has an unusually large heritability component. Results were discussed in terms of their implications for an evolutionary model of animal hypnosis.
\end{abstract}

Tonic immobility, also known as animal hypnosis, is an easily quantifiable phenomenon found in many different species which is induced by a brief period of physical restraint, typically administered by holding an animal down on a flat surface. The state is characterized by profound motor inhibition or cataleptic-like paralysis, intermittent periods of eye closure, changes in heart and respiration rate, altered electroencephalographic patterns, Parkinsonian-like tremors in the extremities, and diminished responsiveness to external stimuli. In different Ss the reaction may last for only a few seconds to over an hour. Figure 1 shows a chicken exhibiting the immobility response.

Tonic immobility seems to represent an unlearned fear reaction (Braud \& Ginsburg, 1973; Gallup, Nash, Potter, \& Donegan, 1970; Maser, Gallup, \& Barnhill, 1973), although many other mechanisms have been postulated (see Ratner, 1967). In terms of its possible ecological significance, immobility may be, or may have been, involved in predator-prey relationships and could represent a terminal defensive reaction in a distance-dependent series of sequential predator defenses (Gallup, Nash, Donegan, \& McClure, 1971; Ratner, 1967). In support of the predator-prey conceptualization, data have been gathered using lizards (Gallup, 1973), chickens (Gallup et al, 1971), and crabs (O'Brien, 1973) which show that different forms of simulated predation increase the duration of tonic immobility. In addition to showing that the reaction can be affected by the threat of predation, support for an evolutionary interpretation of tonic immobility as an evolved predator defense could also be provided by the demonstration of a genetic influence.

To date there has been only one attempt to test for an effect of selective breeding on tonic immobility, which was recently reported by McGraw and Klemm (1973), using rats. After five generations of selective breeding, they obtained statistically reliable differences in the duration of tonic immobility between offspring of parents showing long and brief immobility times. The data, however, remain difficult to interpret since McGraw and Klemm did not include controls for

* The author would like to thank J. L. Mason and N. Woodard for help in breeding and data collection. "maternal effects," in the sense of cross-fostering litters and cross breeding among high and low parents, which are necessary to rule out possible confounding effects of post- and prenatal environmental influences in mammalian species (Broadhurst, 1967).

The present experiment represented an attempt to assess the effects of bidirectional breeding for tonic immobility in domestic chickens.

\section{METHOD}

The original Ss consisted of 80 straight-run Production Red chickens (Gallus gallus) obtained from a local hatchery at 1 day of age. The birds were housed in commercial brooders and

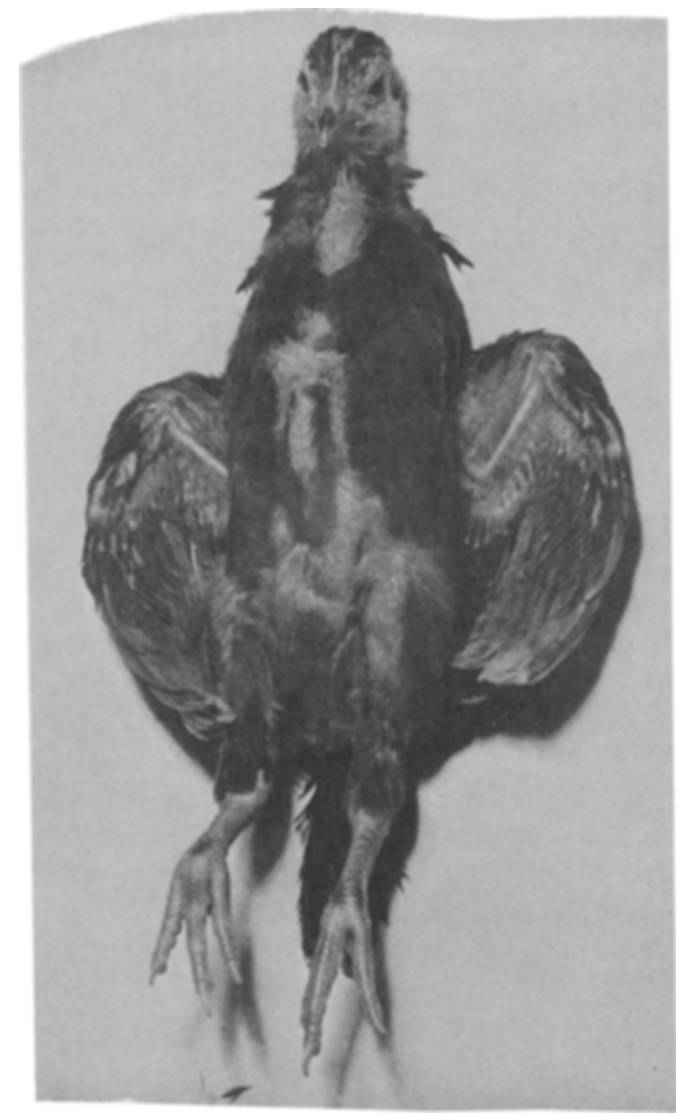

Fig. 1. An immobilized 3-week-old Production Red chicken. 
Table 1

Means and Standard Deviations of the Duration of Tonic Immobility (Seconds) Shown by the Original Population, Parents, and Offspring $\left(F_{1}\right)$

\begin{tabular}{lcccccc}
\hline & $\begin{array}{c}\text { Original } \\
\text { Popula- } \\
\text { tion }\end{array}$ & \multicolumn{2}{c}{ Parents } & & \multicolumn{2}{c}{ Offspring } \\
\cline { 3 - 6 } \cline { 5 - 6 } & High & Low & & High & Low \\
\hline $\mathrm{N}$ & 80 & 6 & 6 & 22 & 45 \\
$\overline{\mathrm{X}}$ & 873.88 & 2568.00 & 61.17 & 2311.45 & 498.36 \\
$\mathrm{SD}$ & 874.07 & 818.24 & 22.89 & & 2517.89 & 841.79 \\
\hline
\end{tabular}

maintained on Purina chick chow (Growena) under conditions of a 14-h photoperiod. At 3 weeks of age all birds were tested for the duration of tonic immobility using $15 \mathrm{sec}$ of manual restraint applied laterally. If a chick failed to show immobility following the first 15-sec induction, successive inductions were given up to a maximum of five. All birds were retained and fitted with plastic leg bands for individual identification after testing.

Eight weeks af ter initial testing, the 10 chickens showing the longest durations of tonic immobility and the 10 showing the shortest reactions at 3 weeks of age were retained and all intermediate birds were discarded. Based on a subjective estimate of vitality, at 5 months of age four birds from each group were again discarded, leaving two males and four females in each. The adults were kept in individual cages and were maintained on Purina Layena chow. Crushed oyster shells were used to supplement the diet provided for hens. Random mating within each group was initiated approximately 2 months after egg laying began and was conducted on a daily to every other day basis. Eggs likewise were marked and collected daily and incubated weekly at $103^{\circ} \mathrm{F}$. Offspring were reared in commercial brooders (Brower, Model B2-6401) containing no less than four and no more than eight brooder mates. The incubators (Brower, Model 11400-3) containing either high or low eggs were randomly varied from 1 week to the next, as were the brooders, so as to provide for equivalent pre- and posthatch environments.

To rule out possible observer bias, the offspring in both groups were tested for the duration of tonic immobility at 3 weeks of age under "blind" conditions by Es who did not know the genetic identity of the Ss. Moreover, birds were all tested under the same conditions, in the same room, and at approximately the same time of day.

\section{RESULTS AND DISCUSSION}

Based on sex determination at 10 weeks of age, no differences were found in the original sample of 80 birds between males and females in the duration of immobility on the initial test, with a point-biserial correlation of only .09, which was nonsignificant. The absence of sex differences is what would be expected from an evolutionary point of view, since as a predator defense there would be little reason for it to be expressed as a dimorphic trait.

Table 1 depicts the major findings in terms of the mean duration of immobility for the original population, parents and offspring. High offspring showed a mean duration of $2311 \mathrm{sec}$, of paralysis as compared to $2568 \mathrm{sec}$ for their parents. Conversely, low offspring showed an average duration of $498 \mathrm{sec}$ as compared to 61 for their parents. Thus, after only one generation, offspring derived from birds showing long immobility times remained immobile an average of $1813 \mathrm{sec}$, or over $30 \mathrm{~min}$ longer than those coming from parents which showed short durations of tonic immobility $(F=19.22$, $\mathrm{df}=1 / 65, \mathrm{p}<.001)$.

Using a linear regression coefficient of offspring on parents, a heritability estimate (Falconer, 1960) of .78 was obtained, using a biserial correlation, with a standard error of \pm .11 . Following a correction for coarse grouping (Wert, Neidt, \& Ahmann, 1954), the heritability estimate increased to .91 , which loosely interpreted means that somewhere between $75 \%$ and $90 \%$ of the trait variance in tonic immobility in this sample is attributable to genetic differences between individuals. Moreover, the effect of selective breeding in opposite directions yielded roughly symmetrical results. After subtracting the most extreme score from each group of offspring and then dividing the response to selection (gain) by the selection differential (reach) in terms of the average immobility times for each of the two groups relative to their parents and the original population, realized heritability estimates of .59 and .58 were found for low and high birds, respectively.

Figure 2 shows the proportion of offspring in each group remaining immobile for varying intervals of time and is another way of illustrating the symmetry. As can be seen, $56 \%$ of low offspring remained immobile for $250 \mathrm{sec}$ or less, while only $9 \%$ of chicks derived from high parents showed reactions that were as brief. On the other hand, $59 \%$ of the high chicks showed responses which lasted over $25 \mathrm{~min}$ and only $4 \%$ of low offspring exhibited responses lasting that long.

Although the use of artificial incubators and brooders would seem to preclude sources of confounding due to differential environmental effects, it could still be argued that the $F_{1}$ differences in duration may have been a reflection of subtle differences in egg chemistry between high and low hens similar to those which may operate prenatally in the mammalian intrauterine environment. As a control for such effects, cross breeding was

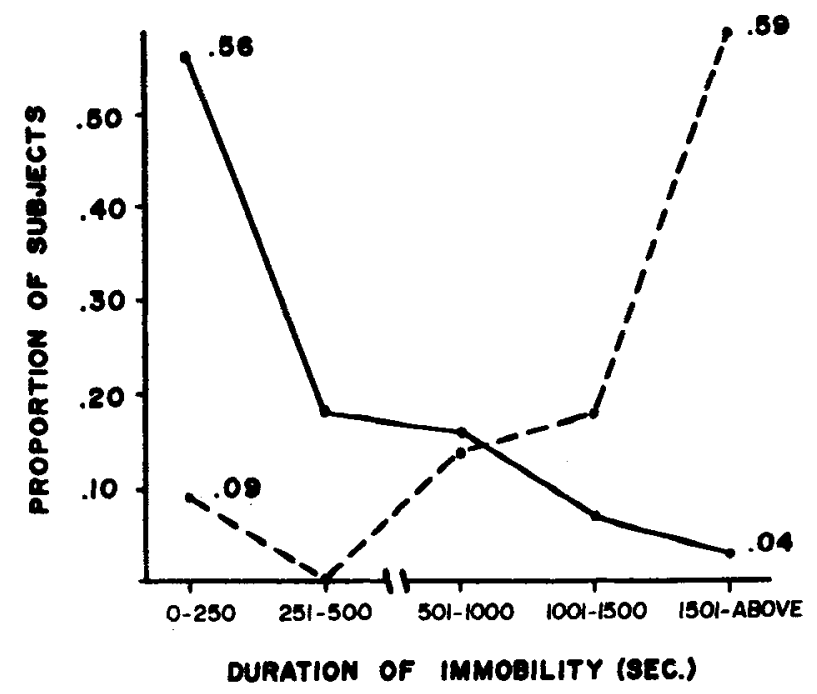

Fig. 2. Proportion of high (dashed line) and low (solid line) offspring remaining immobile for varying intervals of time. 
instituted 3 months later between the two parent populations. As evidence for genetic determination of the original differences, offspring from high $(\mathrm{N}=14)$ and low $(\mathrm{N}=11)$ eggs having low and high paternity, respectively, did not differ significantly in terms of immobility time and showed intermediate reactions averaging about $750 \mathrm{sec}$.

It is interesting to note that, while selective breeding had a pronounced effect on the duration of tonic immobility, there was no difference between high and low offspring in terms of the number of $15-\mathrm{sec}$ inductions needed to elicit the reaction, suggesting that susceptibility, which has been a popular measure of immobility (e.g., Gallup et al, 1971), may be independent of duration. Perhaps an effect on susceptibility to immobility would require selective breeding based on number of inductions rather than on a duration criterion. In agreement, however, with the finding that tonic immobility in rats may be effected by selective breeding (McGraw \& Klemm, 1973), the duration of immobility in chickens does seem to be subject to a genetic influence in the sense that, by manipulating breeding patterns, considerable control could be exercised over the mean expression of the trait.

A final interpretive problem posed by these data relates to the values of the obtained heritability estimates, which are unusually large. In an evolutionary sense, the proportion of trait variance attributable to genetic variability between individuals should be inversely proportional to the degree of natural selection pressure (Falconer, 1960). That is, the genotypic correlates of traits critical for survival should, in the process of being selected for over generations, become more uniformly distributed throughout a population. Therefore, to argue that tonic immobility has survival value as an evolved predator defense (Gallup et al, 1971; Ratner, 1967) would, contrary to the present findings, imply a small heritability estimate. It is important to acknowledge, however, that in the case of the captive domestic chicken immobility does not have survival value but, rather, may have had survival value prior to human intervention. In the process of domestication, continued selection pressure for stabilization of tonic immobility has been greatly diminished, if not eliminated, and the chances of genetic drift correspondingly increased. ${ }^{1}$

A testable behavioral-genetic implication of this interpretation would be that, in general, selective breeding with feral species should yield smaller heritability estimates than with domesticated animals if, as many people suspect, tonic immobility participates in survival and reproductive success under natural conditions.

\section{REFERENCES}

Braud, W. G., \& Ginsburg, H. J. Effect of administration of adrenalin on immobility reaction in domestic fowl. Journal of Comparative \& Physiological Psychology, 1973, 83, 124-127.

Broadhurst, P. L. An introduction to the diallel cross. In J. Hirsch (Ed.), Behavior-genetic analysis. New York: MeGraw-Hill, 1967.

Falconer, D. S. Quantitative genetics. New York: Ronald Press, 1960.

Gallup, G. G., Jr. Simulated predation and tonic immobility in lizards (Anolis carolinensis). Copeia, 1973, 3, 623-624.

Gallup, G. G., Jr., Nash, R. F., Donegan, N. H., \& McClure, M. $K$. The immobility response: A predator-induced reaction in chickens. Psychological R ecord, 1971, 21, 513-519.

Gallup, G. G., Jr., Nash, R. F., Potter, R. J., \& Donegan, N. H. Effect of varying conditions of fear on immobility reactions in domestic chickens (Gallus gallus). Journal of Comparative \& Physiological Psychology, 1970, 73, 442-445.

Maser, J. D., Gallup, G. G., Jr., \& Barnhill, R. Conditioned inhibition and tonic immobility: Stimulus control of an innate fear response in the chicken. Journal of Comparative \& Physiological Psychology, 1973, 83, 128-133.

McGraw, C. P., \& Klemm, W. R. Genetic differences in susceptibility of rats to the immobility reflex ("animal hypnosis"). Behavior Genetics, 1973, 3, 155-161.

O'Brien, T. J. Tonic immobility in the blue crab: Its relation to threat of predation. Unpublished doctoral dissertation, Tulane University, 1973.

Ratner, S. C. Comparative aspects of hypnosis. In J. E. Gordon (Ed.), Handbook of clinical and experimental hyp nosis. New York: Macmillan, 1967.

Wert, J. E., Neidt, C. O., \& Ahmann, J. S. Statistical methods in education and psychological research. New York: Appleton-Century-Crofts, 1954.

\section{NOTE}

1. Genetic drift might also provide an attractive account of the very large individual differences typically found among unselected chickens (note the size of the standard deviation in relation to the mean for the original population in Table 1).

(Received for publication February 26, 1974.) 